\title{
Aux frontières de la sécurité sanitaire. Les controverses métrologiques sur les faibles doses et les perturbateurs endocriniens
}

\author{
Francis Chateauraynaud ${ }^{1}$, Josquin Debaz ${ }^{2}$, Matthieu Fintz ${ }^{3}$ \\ 1 Sociologue, GSPR, EHESS, 75005 Paris, France \\ 2 Historien des sciences, GSPR, EHESS, 75005 Paris, France \\ 3 Politologue, EA 3424, IRIST, Université de Strasbourg, 67085 Strasbourg, France
}

Longtemps, l'analyse des risques s'est appuyée sur le précepte de Paracelse, «c'est la dose qui fait le poison ». L'intérêt de ce paradigme était de donner au gestionnaire la possibilité de définir des seuils de risques acceptables, de concilier usage de produits dangereux et prévention des risques. Comment faire lorsque les effets adverses ne suivent pas ce précepte mais dépendent de nombreux facteurs, fenêtres d'exposition, vulnérabilités individuelles, etc. ? La question est aujourd'hui sur l'agenda avec - notamment - le problème des perturbateurs endocriniens qui bouscule la toxicologie réglementaire. Le texte qui va suivre montre que sur un sujet réputé technique, les recherches en sciences sociales peuvent conduire à poser le problème en des termes nouveaux. Ainsi, les auteurs soutiennent que la véritable question n'est pas celle du changement de paradigme mais celle de l'éventuelle coexistence de plusieurs paradigmes dans un même régime réglementaire.

La Rédaction

\section{Mots-clés :}

santé ;

environnement ; sociologie; faibles doses ; perturbateurs endocriniens
Résumé - La question des faibles doses a déjà une histoire longue, très liée dans un premier temps à la radioactivité, et peut être retracée à travers toute une série de controverses métrologiques à propos de l'évaluation des risques sanitaires et environnementaux. En prenant appui sur les évolutions récentes suscitées par la prise en compte de plus en plus saillante des perturbateurs endocriniens, l'article propose une exploration des différentes logiques épistémiques à l'œuvre dans les milieux de recherche et d'expertise mobilisés autour des faibles doses. À partir d'une analyse documentaire, d'observations participatives de groupes d'experts et d'une série d'entretiens, il examine les appuis cognitifs et techniques utilisés par les acteurs, et les conditions pragmatiques de ce qu'une partie d'entre eux appelle un « changement de paradigme ", conduisant à la redéfinition des concepts et des outils de la toxicologie au XXI ${ }^{\mathrm{e}}$ siècle.

\section{Keywords:}

health; environment; sociology; low doses; endocrine disruptors

\begin{abstract}
At the frontier of health safety: metrological controversies on low doses and endocrine disruptors. The issue of low doses has a long history, initially stemming from radioactivity. It can be followed up through a series of metrological controversies about risk assessment on environmental health issues. Building on recent developments of public policy around the hazards linked to endocrine disruptors, this paper offers an exploration of different epistemic logics shaping research and expertise circles involved in low doses issues. Relying on a literature review, participative observation and a series of interviews with experts, this paper examines cognitive and technical supports used by the experts, and strives to depict the pragmatic conditions of a so-called "paradigm shift", purported to reshape the key concepts and tools of toxicology for the $21^{\text {th }}$ century.
\end{abstract}

Auteur correspondant : F. Chateauraynaud, chateau@msh-paris.fr 
Issues du domaine de la radioprotection, les faibles doses ont marqué durablement les querelles autour du nucléaire, en rapprochant dans un même espace de calcul une logique de seuil et un régime d'acceptabilité des risques (Fagnani et Nicolon, 1979; Boudia, 2007; Bulletin of the Atomic Scientists, 2012). Le suivi des victimes de Hiroshima et de Nagasaki, au cours des années 1950, a servi d'appui à la production de données et à la mise en discussion des questions de seuils. Installant dans le monde de la régulation des risques un modèle de « relation linéaire sans seuil » comme hypothèse par défaut, la Commission internationale de protection radiologique (CIPR) énonce, dans les années 1960, qu'il est impossible de déterminer un seuil en deçà duquel une exposition à des radioéléments est sans danger. À partir de ce modèle, les autorités se sont livrées à différents compromis fondés sur des « seuils d'exposition » et des «doses maximales» en dessous desquels le risque est supposé acceptable ${ }^{1}$. Le principe ALARA (as low as reasonably achievable) a longtemps favorisé ces compromis en servant de base à la régulation des expositions dans la plupart des activités industrielles, y compris autour de la question des pesticides et des additifs alimentaires (Jas, 2007).

De fait, pendant près de trente ans, la question des faibles doses percole discrètement d'un milieu à l'autre, pour revenir en force dans l'espace public au moment des grandes crises sanitaires des années 1990 qui sont aussi marquées par une emprise de plus en plus grande des technologies biomoléculaires. En se plaçant dans la longue durée et en prenant comme indicateur le rapport entre le degré de présence dans la presse ou les débats politiques, d'un côté, et les communications ou publications dans des arènes spécialisées, de l'autre, on observe que la question des faibles doses a pris l'allure d'une cause lancinante, dont les points de saillance restent discrets dans les arènes publiques (Chateauraynaud, 2011). Néanmoins, dans la période récente, les faibles doses sont convoquées dans un nombre croissant de dossiers relevant de la sécurité sanitaire. $\mathrm{Qu}^{\prime} \mathrm{ils}$ reconnaissent la nécessité d'une refonte des protocoles de l'évaluation de risque, d'aucuns annonçant même un "changement de paradigme", ou qu'ils mettent l'accent sur les contraintes interdisciplinaires de la mesure et de la preuve, de nombreux acteurs de la sécurité sanitaire considèrent aujourd'hui les faibles doses dans un nouveau cadre épistémologique.

Cet article présente les résultats d'une enquête menée par le Groupe de sociologie pragmatique et réflexif

\footnotetext{
1 La critique antinucléaire a vu dans la formalisation de cet espace de calcul un modèle de régulation imparfait qui exclut d'office des modèles alternatifs plus favorables à la santé des travailleurs et des populations (Ceri, 2003 ; Thébaud-Mony, 2008).
}

(GSPR) de l'EHESS ${ }^{2}$ en partenariat avec l'Agence nationale de sécurité sanitaire - alimentation, environnement, travail (Anses). L'enquête repose sur une analyse documentaire en santé environnementale (revues, presse, sites Internet) doublée par une quinzaine d'entretiens auprès de chercheurs ayant une activité d'expertise et de responsables d'agences d'évaluation de risque (toxicologues, épidémiologistes, biologistes ou endocrinologues) en France (décembre 2010 - avril 2011) et une dizaine aux États-Unis (septembre - octobre 2010). Par ailleurs, elle s'appuie également sur de multiples observations réalisées à l'occasion de la participation d'un des auteurs (Matthieu Fintz) à un groupe d'experts de l'Anses sur les perturbateurs endocriniens (Chateauraynaud et al., $2011)^{3}$. Pour éclairer les différentes formes de raisonnement des acteurs dans les entretiens et la littérature spécialisée ou dans les argumentations déployées par des groupes d'experts, on a rassemblé les jeux d'arguments dans un espace de variation formé de cinq logiques épistémiques. Notons qu'il ne s'agit pas ici de distinguer des cultures épistémiques (Knorr-Cetina, 1999), même si les différentes combinatoires s'inscrivent bien entendu dans l'histoire de réseaux expérimentaux qui finissent par se cristalliser au fil de la circulation d'entités épistémiques, de modèles, de routines et de savoirs tacites (Rheinberger, 1997). Notre analyse vise plutôt à saisir les chemins par lesquels les acteurs surmontent la complémentarité, l'hétérogénéité ou l'opposition de logiques épistémiques relativement contraignantes.

Dans un premier temps, nous caractérisons des formes de raisonnement dont le déploiement nous est apparu comme une forte condition pragmatique de pertinence des faibles doses : logiques de mesure, de produit à risque, d'effets sanitaires, de mélanges ou codéterminations, et de groupes vulnérables. Oscillant sans cesse entre faits et artefacts, nous montrons, dans une seconde partie, comment la catégorie des perturbateurs endocriniens, issue d'une confrontation des logiques épistémiques et de la recherche d'éléments de preuve, a conduit à réorganiser les discours sur la sécurité sanitaire autour de l'idée d'un « changement de paradigme ».

\section{Les faibles doses et la production de l'incertitude autour des perturbateurs endocriniens}

À l'ère de la précaution, le cas des perturbateurs endocriniens sert de « laboratoire public » pour l'expression des enjeux épistémiques et axiologiques suscités

\footnotetext{
2 École des hautes études en sciences sociales.

3 Des éléments de cette recherche ont également été intégrés dans un programme financé par l'ANR (Boudia, 2011).
} 


\begin{tabular}{|l|l|l|}
\hline $\begin{array}{l}\text { 1. Métrologie : technologies, protocoles } \\
\text { et artifices expérimentaux (ex. toxicologie } \\
\text { analytique, modèles animaux, modèles } \\
\text { cellulaires, biologie des systèmes) }\end{array}$ & $\begin{array}{l}\text { 2. Logique des sources et de facteurs } \\
\text { d'exposition : produits de consommation, } \\
\text { substances chimiques, usages (ex. BPA, } \\
\text { phthalates) }\end{array}$ \\
\hline $\begin{array}{l}\text { 3. Version conséquentialiste des faibles } \\
\text { doses : recherche des causalités à partir de } \\
\text { pathologies et d'effets adverses (ex. cancer } \\
\text { du testicule, obésité, troubles de l'attention) }\end{array}$ & $\begin{array}{l}\text { 4. Dose méréologique et holisme } \\
\text { (ex. mélanges de substances, } \\
\text { écologie et biologie } \\
\text { développementale) }\end{array}$ & \\
\hline
\end{tabular}

Fig. 1. Cinq logiques épistémiques associées au surgissement des faibles doses.

par la montée des alertes sur la présence de produits chimiques dans l'environnement (Myers et al., 2009). Placés au cœur des relations entre contaminants, expositions et réponses des systèmes biologiques, les perturbateurs endocriniens ont de fait atteint ces dernières années un haut degré de visibilité publique, ce dont témoignent différents rapports et avis officiels (Barbier, 2011 ; Anses, 2011). Cette présence médiatique et politique survient après l'enchaînement ininterrompu de crises sanitaires, mais aussi après un long travail d'intéressement mené par des équipes de recherche auprès des pouvoirs publics (agences de financement de la recherche, agences d'évaluation de risque) et des acteurs privés (fondations et sociétés savantes, ONG) depuis les années 1970.

En France, un des points de bifurcation se situe fin 2008 lorsque les pouvoirs publics organisent la mise sur agenda de la question de la fertilité et de l'environnement. Début 2009, les directions générales de la santé et de la prévention des risques saisissent les agences de sécurité sanitaire pour évaluer les risques pour la reproduction de plusieurs dizaines de substances chimiques présentes dans les biens de consommation et les cosmétiques. Parmi elles, le Bisphénol A (BPA) fait figure de substance phare. Omniprésent dans l'environnement et dans les fluides corporels, le BPA est depuis les années 1990 au cœur du jeu politique de la régulation des substances chimiques en Amérique du Nord et en Europe. Alors que, sous la pression des associations de consommateurs et des environnementalistes, les industriels et les distributeurs du secteur agroalimentaire commencent à retirer peu à peu du marché des biens de consommation concernés ${ }^{4}$, des équipes de recherche continuent

\footnotetext{
4 Tout se passe comme si une une régulation « par le marché » avait précédé la régulation publique, créant une série d'incitations à la substitution. Pour des exemples récents: Marc Gunther, "How Wal-Mart became the new FDA », 16 juillet 2008 (http://www.marcgunther.com/2008/07/16/how-walmart-became-the-new-fda/) et « Tupperware retirelebisphenol A de ses produits ", Le Parisien, 8 mai 2012.
}

d'accumuler des données suggérant des effets sanitaires à une exposition inférieure à la dose journalière tolérable (DJT) de $50 \mu \mathrm{g} / \mathrm{kg}$ de poids corporel (vom Saal et al., 2007). Il est remarquable que les récentes évaluations de risque du BPA demandées tant au niveau européen à l'EFSA (European Food Safety Agency) qu'au niveau national français à l'Anses, circonscrivent la question aux effets à "faibles doses " entendus dans ce contexte comme des effets observés à des doses d'exposition inférieures à un seuil réglementaire. C'est précisément cette définition des effets à faibles doses qu'avait retenue en 2002 un panel d'experts formé par le National Toxicology Program (US EPA ${ }^{5}$ ) en parlant de «changements biologiques qui interviennent dans la gamme des expositions humaines ou à des doses plus faibles que celles utilisées typiquement dans le paradigme de tests standards de l'EPA pour évaluer la toxicité pour la reproduction et le développement »(Melnick et al., 2002). Les controverses sur le BPA condensent toutes les variations possibles autour du rôle reconfigurateur des perturbateurs endocriniens. Outre la remise en question de la centralité de la dose et du seuil au profit des vulnérabilités individuelles et la mise en avant des mélanges de substances (Kortenkamp et al., 2009 ; Myers et al., 2009), on observe une forte référence à la durée des expositions et au caractère transgénérationnel des effets (Fig. 1).

Selon une première logique, la faiblesse de la dose est définie par la métrologie utilisée, à partir des instruments déployés dans le cadre confiné du laboratoire. Il s'agit d'une faible dose sous contrainte métrologique au sens où elle renvoie à un contexte expérimental déterminé par des technologies, des protocoles et des artefacts. Du côté de la toxicologie analytique par exemple, tous les acteurs s'accordent à reconnaître l'impact de la diminution des limites de détection qui s'est traduit par la multiplication des substances chimiques mises en évidence dans l'environnement et les organismes, en

\footnotetext{
5 Environmental Protection Agency.
} 
rendant ainsi visibles des phénomènes ignorés auparavant (des pesticides jusqu'à la pollution intérieure). L'expérimentation à faibles doses fondée sur le développement des outils de modélisation a également permis des avancées dans la compréhension du métabolisme des substances chimiques en mettant à jour des taux d'absorption et des voies d'excrétion différents selon la dose. Ce progrès dans la précision instrumentale a élargi le spectre des molécules à détecter, posant ainsi le problème de la hiérarchisation et de l'acceptabilité sociale d'une exposition aux faibles doses. Mais cette plus grande précision et les effets observés sont aussi obtenus au prix d'artefacts dont la pertinence pour l'extrapolation à l'homme est souvent controversée dans les groupes d'experts.

Une deuxième logique conduit les acteurs à se tourner vers les substances, les produits de consommation et les usages. La définition des faibles doses s'inscrit ici dans une logique de sources et de facteurs d'exposition largement déterminée par la protection des consommateurs encadrée par des dispositifs européens comme $\mathrm{REACh}^{6}$. L'émergence des perturbateurs endocriniens est enchâssée dans l'histoire de substances singulières comme le diéthylstilbestrol (Langston, 2010) ou le BPA et de leurs usages alternativement autorisés, interdits ou frauduleux. Selon cette logique, les perturbateurs endocriniens s'inscrivent dans la régulation du marché des substances chimiques et des critères d'évaluation retenus pour une autorisation de mise sur le marché (tonnage, effets critiques évalués). À ce titre, si le règlement REACh offre une porte d'entrée permettant de restreindre ou d'interdire des perturbateurs endocriniens à travers la catégorie des « substances de préoccupation équivalente », l'Union européenne débat toujours de leur définition réglementaire, et partant de leurs critères d'évaluation (Kortenkamp et al., 2011). C'est cette position floue dans les classifications européennes qui fait de la catégorie des perturbateurs endocriniens un enjeu pour toute la régulation des substances chimiques.

Une troisième logique del'espace de variation est celle qui part des conséquences ou des effets visibles - les pathologies ou les effets adverses, dont on suspecte les voies de causalité, depuis les cancers ou les troubles de la reproduction jusqu'aux allergies ou aux formes de l'hypersensibilité (Chateauraynaud et Debaz, 2010). Dans le domaine de l'épidémiologie de la reproduction, les observations faites à partir des registres de cancer des pays scandinaves suggèrent une augmentation de l'incidence de certaines pathologies (cancer du testicule, baisse de la qualité du sperme, cryptorchidie, hypospadias) et

\footnotetext{
6 REACh est un règlement européen entré en vigueur le $1^{\mathrm{er}}$ juin 2007, destiné à encadrer l'enregistrement, l'évaluation et l'autorisation des substances chimiques, ainsi que les restrictions applicables à ces substances.
}

ont, de fait, joué un rôle moteur dans la recherche d'origines environnementales (Jégou et al., 2009). On peut appréhender cette logique comme la version conséquentialiste des faibles doses.

Une des questions majeures posées aux chercheurs est celle de la pertinence des échelles d'observations, depuis les cellules jusqu'aux espèces en passant par l'individu. Du même coup, les protocoles fixant une définition purement métrologique de la dose faible sont souvent opposés à une quatrième logique qui renvoie à l'approche globale d'environnements entremêlés dans lesquels interagissent en permanence toutes sortes d'entités. Une telle approche est au cœur des doctrines développées par l'écologie politique ${ }^{7}$. Elle traverse aussi les approches holistes en médecine (Lawrence et Weisz, 1998) et les débats entre réductionnisme, complexité et causalité dans la biologie contemporaine (Gilbert et Epel, 2009). Donnons-lui le nom de dose méréologique, puisqu'elle introduit au niveau biomoléculaire le monde qui l'a engendrée.

Une cinquième logique conçoit les faibles doses comme le domaine de la variabilité par excellence. Dans ce cadre, le travail du chercheur part d'observations non conventionnelles (comme l'allure non linéaire d'une courbe dose-réponse ou un signalement cellulaire) pour aller vers la caractérisation de l'imprévisible et la mise en évidence de populations vulnérables. Les faibles doses font ici appel à la toxicité intrinsèque d'une substance et à la variabilité interindividuelle des réponses à cette toxicité lorsqu'elle induit des effets chroniques. La variabilité des effets observés à faibles doses est fortement mise en avant par les endocrinologues. Pour appuyer leur propos, ils soulignent le profil complexe des courbes doses-réponses, l'hétérogénéité de populations pourtant génétiquement similaires et la multiplicité des réponses cellulaires et des cibles d'action face à un stress environnemental. Cette hétérogénéité est de plus en plus rapprochée de la variabilité épigénétique, impliquant des mécanismes d'action multiples et plus complexes que la seule mutation de l'ADN. Armés de ces savoirs, les endocrinologues revendiquent une place dans le débat sur les perturbateurs endocriniens et critiquent le réductionnisme de l'approche toxicologique classique (Diamanti-Kandarakis et al., 2009) ${ }^{8}$.

Si cette partition analytique a des vertus pour comprendre les appuis cognitifs des acteurs et les objets de

\footnotetext{
7 Voir « Pour un nouveau paradigme en matière de santé publique, rencontre avec André Cicolella », Agoravox, 14 avril 2011 (http:/ / www.agoravox.fr/actualites/sante/article/pourun-nouveau-paradigme-en-92315).

8 La montée en puissance des sociétés savantes d'endocrinologie se lit par exemple dans $l^{\prime}$ « assistance » qu'elles proposent pour la conduite de l'expertise toxicologique dans la revue Science en 2011 (The American Society of Human Genetics et al., 2011 ; Society of Toxicology et al., 2011).
} 
controverses, ce sont surtout les relations et les entrelacements entre les cinq logiques qui permettent de rendre compte du plus grand nombre d'approches. Par exemple, l'émergence de groupes vulnérables face aux faibles doses s'inscrit aussi dans la logique des sources d'exposition (à travers les produits de consommation destinés à ces groupes) et dans la logique conséquentialiste qui prend appui sur des effets observables suscitant une recherche de causalité (comme l'incidence de cancers du testicule chez l'enfant). Enfin, l'émergence de groupes vulnérables convoque également la logique des technologies, protocoles et artefacts expérimentaux. Ici, il est sans doute essentiel de prendre en compte le déploiement de ces technologies hors du laboratoire suite au développement du marché des tests de prédisposition à certains cancers (Gaudillière et Löwy, 2005), du médicament (Kahn, 2008) et de la médecine personnalisée. Ce qui évoque une forme de «biomolécularisation » $\mathrm{du}$ monde social représente ainsi un puissant facteur d'émergence de groupes vulnérables.

De même, le jeu controversé des expertises montre souvent une opposition entre la logique des sources d'exposition, circonscrites à une évaluation substance par substance pour une voie d'exposition, et la logique méréologique des mélanges ou des cocktails de substances. Cette logique des sources est de plus en plus couplée à une logique de la variabilité par la caractérisation de populations sensibles.

\section{Les faibles doses au regard des risques incertains et de la pertinence toxicologique}

Selon que l'émergence épistémique des faibles doses emprunte les voies de la métrologie, des produits, des effets suspectés, des mélanges ou d'une population vulnérable, son statut peut toujours être soumis à la critique qui l'élève au rang de fait "avéré », "probable» ou «possible», de «biais », $d^{\prime}$ « artefact expérimental» ou de «fraude». Le déploiement des logiques épistémiques conduit par conséquent à penser les faibles doses à partir de définitions contextuelles et de combinatoires différentes de la preuve. Celles-ci font appel aux compétences pragmatiques des experts dont le travail consiste à caractériser les données évaluées et à les hiérarchiser du point de vue de l'incertitude.

Ces combinatoires de la preuve, qui déterminent les façons de considérer le poids de la preuve (weight-ofevidence), peuvent se figer, après d'âpres discussions d'experts, dans des arbres de décision ou des typologies exprimant des degrés d'incertitude sur le caractère avéré ou probable $\mathrm{d}^{\prime}$ un effet suite à une exposition. À l'extrême, ces combinatoires peuvent devenir réglementaires au sens où la réalisation d'un certain nombre de tests selon des conditions méthodologiques très strictes et validées (bonnes pratiques de laboratoire - BPL ; tests réglementaires OCDE) est rendue obligatoire pour les industriels s'ils veulent obtenir le classement et la mise sur le marché de leurs produits 9 .

Parler de «faibles doses » ou de «faibles expositions ", c'est engager d'un côté le rapport entre le visible et l'invisible, ou le « mesurable » et le "non détectable», et de l'autre le rapport entre les situations exceptionnelles, de type accidentel, et les situations d'exposition sur le long terme. Faire ressurgir les faibles doses dans les arènes publiques, c'est en outre réinterroger les garanties épistémiques du système métrologique utilisé par les agences d'évaluation de risque, et partant remettre potentiellement en cause la fiabilité de leurs expertises. À une échelle plus large que le groupe $\mathrm{d}^{\prime}$ experts, chaque nouvelle « menace » mise sur l'agenda des agences de sécurité sanitaire par les pouvoirs publics est suivie par une controverse ou une polémique, dans laquelle des acteurs sèment le doute sur la qualité et la pertinence des données scientifiques évaluées et/ou exclues de l'évaluation de risque. Les expertises sont ainsi jalonnées d'épreuves consistant à mettre en doute la réalité $\mathrm{d}^{\prime} u n$ phénomène.

Deux exemples permettent de mieux saisir ces processus : le rôle de l'épidémiologie dans l'élucidation de l'impact des expositions à faibles doses et la pertinence toxicologique que les experts attribuent aux données évaluées. Dans le domaine des faibles doses, c'est l'épidémiologie qui porte le plus explicitement la tension du doute, car elle se heurte à la distribution quantitative de l'effet critique recherché (cancer, obésité, diabètes, syndromes divers, troubles du comportement et de la cognition) sur un territoire donné. De sérieuses critiques méthodologiques mais aussi éthiques sont ainsi opposées à toute approche transversale qui obtient difficilement des risques significatifs pour des populations faibles. Une controverse de ce type s'est par exemple déployée entre 2008 et 2010 au cour du Circ (Centre international de recherche sur le cancer), suite à la parution d'une critique de la publication de "faux positifs » ou de « faibles associations » en épidémiologie environnementale (Boffetta et al., 2008; Grandjean, 2008; Boffetta et al., 2009 ; Blair et al., 2009 ; Wild et Cogliano, 2010). Certains de ces acteurs en ont appelé à la responsabilité éditoriale des revues face à des publications qui contribueraient à « jeter le discrédit sur la discipline » et, ce faisant, à « dilapider sur des sujets non prioritaires des fonds déjà rares ». En tout état de cause, la question des faux-négatifs et des faux-positifs traverse constamment les débats au sein de l'épidémiologie environnementale.

\footnotetext{
9 Il y aurait sans doute lieu ici de prolonger pour la santé environnementale les travaux réalisés dans le domaine de l'établissement des standards toxicologiques dans le domaine du médicament (Abraham et Reed, 2002).
} 
C'est à travers la prise en compte des limites de l'épidémiologie que les faibles doses sont parfois assimilées à des risques faibles, relativement à des risques solidement établis d'un point de vue quantitatif et relativement bien documentés comme ceux du tabac et de l'alcool. Face aux « limites » de l'épidémiologie, parfois perçues comme indépassables, les experts rencontrés suggèrent de rapprocher la discipline de la situation expérimentale, ce que les chercheurs ne peuvent souvent pas réaliser pour des raisons éthiques et économiques. La mise au point de designs expérimentaux plus facilement contrôlables prend alors appui sur des études de cohortes, respectant à la fois la chronologie des expositions ou des événements de santé au long de la vie et une mesure fondée sur la sélection de biomarqueurs, et non plus seulement sur les expositions déclarées par les sujets. Enfin, les acteurs interrogés rappellent l'importance d'une sélection de populations homogènes afin de mieux contrôler les cofacteurs et les biais.

Un des moteurs des controverses scientifiques sur les effets à faibles doses réside ainsi dans les débats sur la réalité même des phénomènes décrits conduisant soit à leur validation, soit à leur rejet dans les régimes de l'erreur méthodologique, du conflit d'intérêt, voire de la falsification (Wandall et al., 2007). Dans ce cadre, que signifie la pertinence toxicologique à laquelle se heurtent les ONG (en particulier le Réseau environnement santé en France), souvent promptes à dénoncer publiquement les choix d'exclure une partie de la littérature dans les évaluations de risque ? C'est encore de la fabrique de la pertinence toxicologique dont parlent des chercheurs praticiens de l'expertise lorsqu'ils soulignent la tendance des agences à savamment ignorer certaines données publiées par la recherche académique, au profit de la seule référence à la toxicologie réglementaire ou à des " principes universels de la toxicologie ».

La fabrique de la pertinence toxicologique est le produit de l'évaluation de la littérature scientifique par un groupe d'experts. Cette évaluation prend en compte la méthodologie et la qualité des données (nombre de doses, randomisation, etc.), l'impact du dispositif expérimental (comme le mode d'administration de la dose ou le régime alimentaire des animaux de laboratoire) sur les résultats observés, l'effet adverse retenu et sa pertinence biologique, le caractère reproduit ou non de ces données, et enfin l'échelle biologique de l'étude (in vitro, in vivo, épidémiologie). Typiquement, les experts sont confrontés aux effets du changement d'échelle, du niveau subcellulaire au niveau de l'organisme entier, et aux contraintes de transposition des modèles animaux au modèle humain. Dans l'expression du scepticisme face aux effets des faibles doses, certains experts ne remettent pas en cause la plausibilité biologique de tel ou tel mécanisme d'action, mais soulèvent le manque général de réplication des études ou s'interrogent sur les différences développementales entre l'animal de laboratoire et l'homme. Du même coup, la question de la reproductibilité et des conditions sociales nécessaires à la réplication fait continûment l'objet de discussions. Une conjoncture de crise sanitaire ou l'existence de procédures judiciaires favorise les études de reproductibilité tandis que l'absence d'originalité d'un résultat reproduit expérimentalement tend à réduire les possibilités de publication dans les revues.

\section{Trois « paradigmes » en tension}

Alimentées par la confrontation des logiques épistémiques et de la recherche d'éléments de preuve, les disputes autour des faibles doses et des perturbateurs endocriniens sont marquées par une référence constante à un "changement de paradigme». Utilisée par les acteurs eux-mêmes, cette référence est assez proche de la définition kuhnienne (Kuhn, 1983) et de son modèle de rupture dans les sciences : les observations d'effets aux faibles doses feraient figure d'anomalie déstabilisant la doctrine toxicologique de référence, laquelle reste fondée à la fois sur le précepte de Paracelse ${ }^{10}$, l'évaluation substance par substance, la sélection d'un mécanisme $\mathrm{d}$ 'action et le recours exclusif au modèle animal.

Parler de "changement de paradigme" revient d'abord à accréditer un processus de changement, de marquer la différence et de désigner des points de rupture face à une expérience passée. Certains experts rappellent bien l'ancrage de leur parcours dans l'expertise des produits chimiques depuis les années 1970 tant dans leur propre pays qu'au niveau transnational et dans l'expérience qu'ils en ont acquise. La trajectoire de certains d'entre eux est fortement mêlée à l'histoire de la régulation de substances à risque spécifiques, à l'instar du mercure pour l'épidémiologiste danois Philippe Grandjean (Grandjean, 2008) comme le sont les PCB, les retardateurs de flamme et le bisphénol A pour d'autres. $C^{\prime}$ est souvent au regard de cette histoire marquée par la lente diminution des seuils réglementaires et par l'émergence de nouveaux effets critiques (reproduction, troubles neurologiques) qu'ils justifient leur engagement pour intégrer la prise en compte des signaux précoces dans l'évaluation de risque. Aux États-Unis, la montée en puissance de réformateurs de la science réglementaire autour des perturbateurs endocriniens a été décrite par Sheldon Krimsky (2000) qui rappelle le rôle structurant joué par l'histoire des organochlorés dans ce pays.

Fortement ancrés dans la pratique de la recherche au sein d'équipes et d'universités prestigieuses, ces réformateurs animent des manifestations scientifiques (par

\footnotetext{
10 «Toutes les choses sont poison, et rien n'est sans poison; seule la dose fait qu'une chose n'est pas un poison. »
} 
exemple, les Prenatal Programming and Toxicology Conferences organisées depuis 2007) se concluant souvent par une déclaration collective (scientific statement), publient revues de la littérature (Vandenberg et al., 2012) et papiers originaux, siègent dans des groupes d'experts européens ou américains, et bénéficient du soutien de fondations (l'Endocrine Disruption Exchange fondé par la naturaliste et environnementaliste Theo Colburn aux États-Unis ou le Collège Ramazzini en Europe). Certains d'entre eux entretiennent également des relations continues avec les ONG de santé environnementale (par exemple, Artac et le Réseau environnement santé en France les invitent régulièrement lors d'événements mobilisateurs).

Mais le discours sur le " changement de paradigme » ne saurait se limiter à un discours porté par un groupe homogène de réformateurs de la science réglementaire. Il est en fait beaucoup plus vaste en raison des combinaisons multiples entre les cinq logiques épistémiques présentées ci-dessus. C'est ici que la fonction argumentative du registre du paradigme dans les controverses mérite d'être précisée. En fait, les entretiens, les observations et la recherche documentaire font apparaître trois " paradigmes » différents auxquels les acteurs peuvent alternativement faire référence lorsqu'ils parlent de faibles doses et de perturbateurs endocriniens. Ces «paradigmes » sont loin d'être homogènes entre eux (ils peuvent ne pas aller dans la même direction) et dans leur structure même (se situer à l'intérieur d'un "paradigme » ne suppose pas de s'entendre au préalable sur des critères robustes pour l'évaluation de risque). Pour autant, ces trois "paradigmes » sont autant de façons d'articuler les cinq logiques épistémiques dans la critique du " paradigme du seuil », dans la promotion d'un «paradigme des origines fœetales » des maladies témoignant de l'importance de la durée des expositions et des populations vulnérables, enfin dans la redéfinition de la notion d'effet adverse, conséquence du "paradigme de la toxicologie du XXI ${ }^{\mathrm{e}}$ siècle». Dans cette optique, les perturbateurs endocriniens et les faibles doses apparaissent comme des objets et des concepts assez peu stabilisés dans l'usage courant mais plus clairement spécifiés dans les contextes expérimentaux (Star et Griesemer, 1989 ; Löwy, 1990) dont la circulation et la mobilisation dans les controverses permettent aux acteurs de définir les limites de l'évaluation scientifique des risques. Autrement dit, ils servent avant tout à articuler, dans un sens ou dans l'autre, un discours sur la sécurité sanitaire.

\section{La remise en cause du " paradigme du seuil »}

Parfois vécue comme un corollaire de l'affinement des instruments métrologiques, la tendance à la diminution des seuils réglementaires répond aussi, d'après les experts, à une diminution de l'acceptabilité sociale de certains risques dans les sociétés contemporaines. Inversement, si la tendance à la diminution des seuils est parfois critiquée par une partie d'entre eux parce qu'elle contraint à aller au-delà de ce que montrent les «faits scientifiques", ils reconnaissent aussi que la fixation d'un seuil répond à un impératif social de production et d'innovations économiques dépendant de l'usage de substances à risque. Avec les perturbateurs endocriniens, la pertinence même de l'idée de seuil est interrogée par une partie de la communauté scientifique en raison de la variabilité des réponses biologiques et de la multiplicité des cibles.

Doublant la critique du «paradigme du seuil», la question des mélanges de substances sous-tend non seulement la prise en compte de multiples expositions là où des seuils ont été fixés substance par substance, mais aussi l'idée que quelque chose de plus que la simple addition des substances peut se produire. Les notions de synergie et de potentialisation symbolisent cette crainte d'une émergence incontrôlée. Si la caractérisation des mélanges fait son chemin dans les laboratoires et dans les agences sanitaires, les données produites font encore l'objet de vives controverses (Arnold et al., 1996; McLachlan, 1997; Marty et al., 2011) et les experts rencontrés préfèrent la prudence dans la référence à des effets synergiques et potentialisateurs.

\section{Le " paradigme des origines fœtales des maladies »}

Tous les acteurs s'accordent à reconnaître l'importance prise par la durée dans l'étude des pathologies contemporaines et l'évaluation de risque. Parmi les cinq points soulevés par l'Endocrine Society sur les perturbateurs endocriniens, trois font référence à la «durée » : l'âge au cours de l'exposition (fenêtre de sensibilité), la latence entre l'exposition et l'effet, et les effets transgénérationnels par des mécanismes épigénétiques (DiamantiKandarakis et al., 2009). À ces trois catégories de durée, on peut ajouter celle de l'exposition chronique tout au long de la vie. Pour les ONG s'appuyant sur la littérature scientifique, on serait passé d'un modèle dans lequel la « dose fait le poison » à un modèle selon lequel c'est la « durée qui fait le poison ».

Rien ne met plus en évidence le caractère désormais incontournable de la durée que la question de l'exposition fœtale. L'intérêt suscité pour les expositions fœtales aux substances chimiques renvoie ainsi, dans la littérature, à un véritable paradigme, pour lequel tout reposerait sur la « plasticité développementale » du fœtus face aux déterminants environnementaux et sociaux, faisant de la période fotale la période de susceptibilité par excellence. Des mécanismes d'action épigénétiques expliqueraient cette plasticité compliquant ainsi le discours public sur les déterminants " génétiques », d'abord définis comme des modifications du génome (quête 
scientifique et pharmacologique des polymorphismes génétiques), mais incluant désormais des modifications de l'expression de certains gènes (phénotype) à une échelle temporelle beaucoup plus courte que celle de l'évolution. Dans une certaine mesure, l'épigénétique raccourcit le temps nécessaire (effets transgénérationnels) pour que des déterminations environnementales s'impriment sur le matériel génétique.

L'hypothèse des origines fœales des maladies est issue de l'épidémiologie et, en particulier, de l'épidémiologie de la nutrition au Royaume-Uni, sans rapport quelconque avec la notion de dose. Dans les années 1980, une équipe d'épidémiologistes propose l'hypothèse d'une relation de causalité entre le faible poids mesuré à la naissance et le développement de pathologies ou de facteurs de risque comme l'obésité, les maladies cardiovasculaires, et la pression artérielle à l'âge adulte. Connue sous le nom de Barker Hypothesis (Barker et al., 1989), cette association entre vie fœetale et maladies à l'âge adulte a produit des travaux se situant bien au-delà des frontières de l'épidémiologie de la nutrition. Si l'écho de l'étude de Barker résonne aussi loin, c'est dû à sa temporalité particulière : il s'agissait en effet d'étudier une cohorte d'individus nés en 1946 et suivis jusqu'à nos jours, et dont la revue Nature a célébré le $65^{\mathrm{e}}$ anniversaire en 2011 (Pearson, 2011).

Les débats actuels sur les perturbateurs endocriniens ont ainsi des ramifications lointaines. Les remous qu'ils provoquent dans le monde de la sécurité sanitaire renvoient aux débats qui agitent les sciences de l'évolution à la recherche d'une nouvelle «synthèse » au regard des mécanismes épigénétiques de transmission des caractères acquis (Gissis et Jablonka, 2011). Pour certains experts en effet, l'émergence des perturbateurs endocriniens traduit un retour du néo-lamarckisme dans la compréhension des phénomènes biologiques.

\section{La biomolécularisation de la toxicologie et la redéfinition des effets adverses : le " paradigme de la toxicologie pour le $\mathrm{XXI}^{\mathrm{e}}$ siècle »}

Comme le souligne le biologiste de l'évolution Scott Gilbert, souvent convoqué par les Bisphenol A Scientists américains, "il est ironique que les outils moléculaires les plus réductionnistes et les plus analytiques aient abouti à montrer la nécessité, et en fait la réalité, de la transmission douce qui avait été exclue de l'étude de l'évolution » (Gilbert, 2011). De manière similaire, les controverses sur la définition des perturbateurs endocriniens et sur leurs effets sont continuellement alimentées par le développement d'outils biomoléculaires de screening soumis à la validation et à leur intégration dans l'arsenal des tests réglementaires.

Dans ce contexte, le rapport Testing Toxicity du National Research Council (NRC, 2007) a joué un rôle majeur dans la promotion de ce que les acteurs de l'évaluation de risque ont nommé un nouveau "paradigme pour la toxicologie du XXI ${ }^{\mathrm{e}}$ siècle». Ce nouveau paradigme repose sur l'usage massif des méthodes in vitro intégrées dans le screening haut débit de substances chimiques dont on cherche à caractériser le parcours toxicologique dans l'organisme à travers la détection d'effets critiques précurseurs (Boekelheide et Campion, 2010). Il s'agit là véritablement d'une «vision » de l'avenir orientant dès à présent les stratégies de test et, en particulier, celle de l'Endocrine Disruptors Screening Program de l'EPA pour la première phase de sélection et de hiérarchisation des perturbateurs endocriniens aux États-Unis. Dans l'Union européenne, la régulation des cosmétiques et la promotion du bien-être animal dans l'expérimentation des substances chimiques représentent également un puissant levier pour la validation des tests in vitro.

Dans ce nouveau "paradigme», il est beaucoup question de la réorganisation des mondes de la toxicologie autour d'une tension entre la clinique et le modèle animal, d'un côté, et la molécularisation de la toxicologie, de l'autre. Plusieurs interlocuteurs signalent ainsi la différence de points de vue entre une toxicologie inspirée de la clinique, héritière des enseignements donnés dans les écoles vétérinaires, et une toxicologie des systèmes, résultat des transformations de la biologie contemporaine. L'arrivée des outils biomoléculaires dans la toxicologie semble bousculer les équilibres sociaux préexistants entre écotoxicologie, toxicologie animale et toxicologie humaine en contraignant à une approche interdisciplinaire.

Le rôle joué par les perturbateurs endocriniens dans cette reconfiguration ne tient pas du hasard : en ciblant des mécanismes d'action plus subtils et plus latents que la génotoxicité, les perturbateurs endocriniens attirent le regard vers des mécanismes causaux qui ne sont pas appréhendés par les tests toxicologiques réglementaires. Partant, c'est la manière de définir le pathologique et la maladie elle-même (le cancer, l'obésité, les troubles reprotoxiques) qui est rediscutée. Pour certains auteurs (Marty et al., 2011), le changement de "paradigme " ne proviendrait pas tant des effets adverses observés à des faibles doses de toxiques ciblant le système endocrinien. Sur le plan de la preuve des effets (cancer du sein et déclin de la production spermatique sont les deux exemples choisis), ces auteurs ont plutôt tendance à penser que les premières alertes ont été infirmées. Pour eux, le «changement de paradigme " provient surtout de la nouvelle manière de définir le caractère adverse des effets de l'exposition aux perturbateurs endocriniens : là où certains experts appréhenderont l'expression d'une protéine comme un changement biologique qui n'a pas forcément un grand pouvoir de prédiction en termes d'effets sanitaires, d'autres y verront des signaux précoces suffisamment préoccupants pour sonner l'alerte. 
On le voit, les appropriations du «paradigme de la toxicologie du XXI $\mathrm{XI}^{\mathrm{e}}$ siècle » sont loin d'être homogènes ${ }^{11}$. Certains de ses promoteurs sont assez peu enclins à accréditer l'idée d'effets sanitaires à faibles doses. Par exemple, Thomas Hartung, ancien directeur de l'ECVAM (European Center for the Validation of Alternative Methods) annonce une toxicologie fondée sur la preuve (evidence-based toxicology), armée de méthodes alternatives à l'expérimentation animale dont il se fait le pourfendeur. Pour autant, sa pragmatique de l'incertitude relève davantage du modèle classique de la relation linéaire avec seuil et de la chasse aux fauxpositifs que d'une croisade contre les substances à faible dose (Hartung, 2011).

Pour plusieurs toxicologues rencontrés, la recherche de manifestations de plus en plus précoces fait perdre de vue la notion d'effet adverse définie par l'anatomopathologie animale. Les études in vitro sur les mécanismes d'action leur semblaient de peu d'intérêt pour l'évaluation de risque. Cette réticence se double pour certains d'un «fossé » entre la recherche académique et le monde de l'évaluation des risques. Orientés par des critères d'évaluation différents entre ces deux mondes, les chercheurs «poussent » leur recherche vers l'étude de mécanismes d'action, pour des raisons de publication, là où l'évaluateur de risque aurait besoin de caractériser un effet adverse cliniquement défini. De ce point de vue, les praticiens de l'évaluation de risque font part de difficultés à traiter une littérature hétérogène, depuis les études normées de l'évaluation réglementaire jusqu'aux études de recherche fondamentale, en passant par une multitude d'études aux protocoles et objectifs mixtes.

Pour d'autres toxicologues, il faut au contraire prendre au sérieux les effets biologiques observés à des stades sublétaux en ce qu'ils peuvent «annoncer» ou favoriser des pathologies ultérieures. Un des arguments avancés pour justifier cette approche a été de mettre en avant la spécificité des processus étudiés à faibles doses là où la toxicologie des fortes doses « inonde » l'organisme de toxiques et ne permet pas de caractériser des réponses biologiques qui touchent plusieurs cibles et tissus simultanément, à la manière des perturbateurs endocriniens.

\section{Conclusion}

Au-delà des controverses savantes et des conflits d'expertise qui divisent, pour longtemps encore, les

\footnotetext{
11 À plus forte raison si on considère la biologie des systèmes dans toutes ses applications en allant jusqu'à la biologie synthétique (Calvert et Fujimura, 2011).
}

champs scientifiques et institutionnels, l'ouverture à une pluralité de régimes épistémiques semble s'imposer dans le paysage de l'expertise sanitaire et environnementale. Comme pour la santé au travail, dont certains aspects sont passés de l'état d'expériences singulières à l'état d'instruments de mesure -comme le stress ou le suicide au travail -, les systèmes experts finissent par incorporer dans leurs espaces de calcul l'expression publique des préoccupations des personnes et des groupes aux prises avec le monde sensible. L'introduction des problèmes endocriniens, des nouvelles relations entre toxicologie et épidémiologie, des cocktails de substances, des populations vulnérables, des fenêtres de sensibilité ainsi que de la durée des expositions correspondent à une "ré-endogénéisation » des alertes et des discussions publiques au cœur des pratiques et des dispositifs de la recherche et de l'évaluation des risques. Dans ce processus, les points de controverse autour de la notion de faibles doses agissent comme des opérateurs de reconfiguration des rapports entre recherche fondamentale, évaluation des risques et gestion publique des vulnérabilités. Si de nouvelles visions du futur de la toxicologie sont déjà performatives, il n'est pas sûr que les critères pour en définir et en standardiser les procédures soient $\mathrm{d}^{\prime}$ ores et déjà fixés. On peut dès lors s'attendre à des rebondissements permanents, sans doute nécessaires à l'exercice de la vigilance collective face aux menaces les plus invisibles.

Les auteurs remercient l'ensemble des chercheursexperts et des responsables des agences et instituts de sécurité sanitaire qui ont accepté de les recevoir.

\section{Références}

Abraham, J., Reed, T., 2002. Progress, innovation and regulatory science in drug development: The politics of international standard-setting, Social Studies of Science, 32, 3, 337-369.

Anses, 2011. Effets sanitaires du bisphénol A et Connaissances relatives aux usages du bisphénol $A$. Rapports de l'Anses.

Arnold, S.F., Klotz, D.M., Collins, B.M., Vonier, P.M., Guillette Jr., L.J., McLachlan, J.A., 1996. Synergistic activation of estrogen receptor with combinations of environmental chemicals, Science, 272, 1489-1492.

Barbier, G., 2011. Perturbateurs endocriniens, le temps de la précaution. Rapport de l'Office parlementaire d'évaluation des choix scientifiques et technologiques, 765.

Barker, D.J., Winter, P.D., Osmond, C., Margetts, B., Simmonds, S.J., 1989. Weight in infancy and death from ischaemic heart disease, Lancet, 9, 577-580.

Blair, A., Saracci, R., Vineis, P., Cocco, P., Forastiere, F., Grandjean, P., Kogevinas, M., Kriebel, D., McMichael, A., Pearce, N., Porta, M., Samet, J., Sandler, D.P., Seniori Costantini, A., Vainio, H., 2009. Epidemiology, public health and the rhetoric of false positives, Environmental Health Perspectives, 2009, 117, 12, 1809-1813. 
Boekelheide, K., Campion, S. N., 2010. Toxicity testing in the 21st century: Using the new toxicity testing paradigm to create a taxonomy of adverse effects, Toxicological Sciences, 114, 1, 20-24.

Boffetta, P., McLaughlin, J.K., La Vecchia, C., Tarone, R.E., Lipworth, L., Blot,W.J., 2008. False-positive results in cancer epidemiology: A plea for epistemological modesty, Journal of the National Cancer Institute, 100, 988-995.

Boffetta, P., McLaughlin, J.K., La Vecchia, C., Tarone, R.E., Lipworth, L., Blot,W.J., 2009. Re: False-positive results in cancer epidemiology: A plea for epistemological modesty, Journal of the National Cancer Institute, 101, 3, 213-14.

Boudia, S., 2007. Naissance, extinction et rebonds d'une controverse scientifique: les dangers de la radioactivité pendant la guerre froide, Mil Neuf Cent, Revue d'Histoire Intellectuelle, 25, 157-170.

Boudia, S., 2011. Les cadres de l'expertise à l'épreuve des expositions aux faibles doses. Programme ANR 2008-2011.

Bulletin of the Atomic Scientists, 2012. Special issue on the risks of exposure to low-level radiation, 68, 3, 10-83.

Calvert, J., Fujimura, J.H., 2011. Calculating life? Duelling discourses in interdisciplinary systems biology, Studies in the History and Philosophy of Biological and Biomedical Life Sciences, 42, 155-163.

Ceri, 2003. Étude des effets sanitaires de l'exposition aux faibles doses de radiation ionisante à des fins de radioprotection. Recommandations du Comité européen sur le risque de l'irradiation, Paris, Frison-Roche.

Chateauraynaud F., 2011. Argumenter dans un champ de forces: essai de balistique sociologique, Paris, Pétra.

Chateauraynaud F., Debaz J., 2010. Le partage de l'hypersensible : le surgissement des électro-hypersensibles dans 1'espace public, Sciences Sociales et Santé, 28, 3, 5-33.

Chateauraynaud F., Debaz J., Fintz M., 2011. La dose fait-elle toujours le poison? Une analyse sociologique des mondes de la recherche et de l'expertise à l'épreuve des faibles doses, document de recherche Anses/GSPR (http:// gspr.ehess.free.fr/documents/rapports/RAP-2011-ANSESFADO.pdf, consulté le 06/09/13).

Diamanti-Kandarakis E., Bourguignon, J.P., Giudice, L.C., Hauser, R., Prins, G.S., Soto, A.M., Zoeller, R.T., Gore, A.C., 2009, Endocrine-disrupting chemicals: An Endocrine Society scientific statement, Endocrine Reviews, 30, 4, 293-342.

Fagnani, F., Nicolon, A., 1979. Nucléopolis : matériaux pour l'analyse d'une société nucléaire, Grenoble, Presses Universitaires de Grenoble.

Gaudillière, J.-P., Löwy, I., 2005. Science, markets and public health: Contemporary testing for breast cancer predispositions, in Berridge V., Loughin K. (Eds), Medicine, the Market and the Mass Media: Producing Health in the Twentieth Century, London, Routledge, 266-87.

Gilbert, S., Epel, D., 2009. Ecological Developmental Biology: Integrating Epigenetics, Medicine and Evolution, Sunderland (MA), Sinauer Associates Inc.

Gilbert, S., 2011. The decline of soft inheritance, in Gissis, S., Jablonka, E. (Eds), Transformations of Lamarckism: From Subtle Fluids to Molecular Biology, Cambridge (MA), MIT Press, 121-125.
Gissis, S., Jablonka, E. (Eds), 2011. Transformations of Lamarckism: From Subtle Fluids to Molecular Biology, Cambridge (MA), MIT Press.

Grandjean, P., 2008. Seven deadly sins of environmental epidemiology and the virtues of precaution, Epidemiology, 19, 1, 158-162.

Grandjean, P., Perez, M., 2008. Developmental neurotoxicity: Implications of mercury research, International Journal of Environment and Health, 2, 3-4, 417-428.

Hartung, T., 2011. From alternative methods to a new toxicology, European Journal of Pharmaceutics and Biopharmaceutics, 77, 3, 338-349, doi:10.1016/j.ejpb.2010.12.027.

Jas, N., 2007. Public Health and pesticide regulation in France before and after Silent Spring, History and Technology, 23, 4, 369-388.

Jégou, B., Jouannet, P., Spira, A., 2009. La fertilité est-elle en danger?, Paris, La Découverte.

Kahn, J., 2008. Exploiting race in drug development: BiDil's interim model of pharmacogenomics, Social Studies of Science, 35, 5, 737-758.

Knorr-Cetina, K.D., 1999. Epistemic Cultures: How the Sciences Make Knowledge, Cambridge (MA), Harvard University Press.

Kortenkamp, A., Backhaus, T., Faust, M., 2009. State of the Art Report on Mixture Toxicity. Report for Directorate General for the Environment of the European Commission.

Kortenkamp, A., Martin, O., Faust, M., Evans, R., McKinlay, R., Orton, F., Rosivatz, E., 2011. State of the Art Assessment of Endocrine Disrupters: Final Report. Report to the Commission of the European Union (http:/ / ec.europa.eu/environment/endocrine/documents/4_SOTA\%20EDC\%20Final\% 20Report\%20V3\%206\%20Feb\%2012.pdf, consulté le 06/ 09/13).

Krimsky, S., 2000. Hormonal Chaos: The Scientific and Social Origins of the Environmental Endocrine Hypothesis, Baltimore, John Hopkins University Press.

Kuhn, T.S., 1983. La Structure des révolutions scientifiques, Paris, Champs Flammarion.

Langston, N., 2010. Toxic Bodies: Hormone Disruptors and the Legacy of DES, New Haven, Yale University Press.

Lawrence, C., Weisz, G. (Eds), 1998. Greater Than the Parts: Holism in Biomedicine, 1920-1950, New York, Oxford University Press.

Löwy, I., 1990. The strength of loose concepts - boundary concepts, federative experimental strategies and disciplinary growth: The case of immunology, History of Science, 30, 90, 371-396.

Marty, M.S., Carney, E.W., Rowlands, J.C., 2011. Endocrine disruption: Historical perspectives and its impact on the future of toxicology testing, Toxicological Sciences, 120(S1), S93-S108.

McLachlan, J. A., 1997. Synergistic effect of environmental estrogens: Report withdrawn, Science, 277, 462-463.

Melnick, R., Lucier, G., Wolfe, M., Hall, R., Stancel, G., Prins, G., Gallo, M., Reuhl, K., Ho, S.-M., Brown, T., Moore, J., Leakey, J., Haseman, J., Kohn, M., 2002. Summary of the National Toxicology Program's report of the endocrine 
disruptors low-dose peer review, Environmental Health Perspectives, 110, 4, 427-431.

Myers, J.P., Zoeller, R.T., vom Saal, F.S., 2009. A clash of old and new scientific concepts in toxicity, with important implications for public health, Environmental Health Perspectives, 117, 11, 1652-1655, doi:10.1289/ehp.0900887.

NRC, 2007. Testing Toxicity in the 21st Century: A Vision and a Strategy, Washington, The National Academy Press.

Pearson, H., 2011. Study of a lifetime, Nature, 471, 20-24.

Rheinberger H.-J., 1997. Towards a History of Epistemic Things: Synthesizing Proteins in the Test Tube, Stanford (CA), Stanford University Press.

Society of Toxicology, Environmental Mutagen Society, Teratology Society, 2011. Toxicologists welcome assessment assistance, Science, 332, 536.

Star, S. L., Griesemer, J., 1989. Institutional ecology, "translations", and boundary objects: Amateurs and professionals in Berkeley's museum of vertebrate zoology, 1907-1939, Social Studies of Science, 19, 387-420.

The American Society of Human Genetics, The American Society for Reproductive Medicine, The Endocrine Society, The Genetics Society of America, The Society for Developmental Biology, The Society for Pediatric Urology, The Society for the Study of Reproduction, and The Society for
Gynecologic Investigation, 2011. Assessing chemical risk: Societies offer expertise, Science, 331, 1136.

Thébaud-Mony, A., 2008. Travailler peut nuire gravement à votre santé, Paris, La Découverte.

Vandenberg, L. N., Colborn, T., Hayes, T.B., Heindel, J.J., Jacobs Jr., D.R., Lee, D.-H., Shioda, T., Soto, A.M.,vom Saal, F.S.,Welshons, W.V., Zoeller, R.T., Myers, J.-P., 2012. Hormones and endocrine-disrupting chemicals: Low-dose effects and nonmonotonic dose responses, Endocrine Reviews, 33, 3 (http://edrv.endojournals.org/content/ early/2012/03/14/er.2011-1050.abstract, consulté le 06/ 09/13).

Vom Saal, F.S., Akingbemi, B.T., Belcher, S.M., Birnbaum, L.S., Crain, D.A., Farabollini, F., Guillette, L.J., Hauser, R., Heindel J.J., Ho, S-M., 2007. Chapel hill Bisphenol A panel consensus statement: Integration of mechanisms, effects in animals and potential to impact human health at current levels of exposure, Reproductive Toxicology, 24, 2, 131-138.

Wandall, B., Hansson, S.O., Rudén, C., 2007. Bias in toxicology, Archives in Toxicology, 81, 605-617.

Wild, C., Cogliano, V., 2010. Letter to the editor: A plea on behalf of expert evaluation and the experts involved, International Journal of Epidemiology, 1, 1, doi:10.1093/ije/ dyq038. 